International Journal of Modern Physics A, Vol. 16, No. 13 (2001) 2447

(c) World Scientific Publishing Company

\title{
ERRATA \\ WHICH CAUSALITY? DIFFERENCES BETWEEN TRAJECTORY AND COPENHAGEN ANALYSES OF AN IMPULSIVE PERTURBATION
}

\author{
[INT. J. MOD. PHYS. A, Vol. 14, No. 7 (1999) 1111-1124] \\ EDWARD R. FLOYD \\ 10 Jamaica Village Road, Coronado, California 92118-3208, USA \\ E-mail: floyd@crash.cts.com
}

The author wishes to make the following amendments:

The evaluation of the matrix element $(\Delta V)_{00}$ is corrected. This correction increases the differences between the trajectory and Copenhagen analyses.

The unnumbered displayed equation preceding Eq. (9) is corrected by including the factor $\delta(t-\tau) / q$ so that $(\Delta V)_{00}$ becomes $^{1}$

$$
(\Delta V)_{00}=\langle 0|\Delta V| 0\rangle=\frac{\delta(t-\gamma)}{q}\left[\frac{\epsilon^{2}}{2}-\frac{1}{4 k^{2}}+\frac{\cos (2 k \epsilon)}{4 k^{2}}\right] .
$$

Consequently, the correct first-order change in energy, $E_{1}$, of an ensemble of particles is given by correcting Eq. (9) to read

$$
E_{1}=F(\Delta V)_{00}=\frac{F \delta(t-\gamma)}{q}\left[\frac{\epsilon^{2}}{2}-\frac{1}{4 k^{2}}+\frac{\cos (2 k \epsilon)}{4 k^{2}}\right] .
$$

Including this correcting factor $\delta(t-\tau) / q$ increases the magnitude of $E_{1}$ during perturbation for the Copenhagen interpretation. Meanwhile, the average $E_{1}$ of the ensemble of particles for the trajectory representation under Copenhagen epistemology remains $\left\langle E_{1}\right\rangle_{\text {average }}=0$ during perturbation. ${ }^{1}$ This strengthens our findings that trajectory and Copenhagen analyses differ regarding perturbing impulses.

\section{References}

1. E. R. Floyd, Int. J. Mod. Phys. A14, 1111 (1999). 